\title{
Invariance of the correlation energy at high density and large dimension in two-electron systems
}

\author{
Pierre-François Loos* and Peter M. W. Gill ${ }^{\dagger}$ \\ Research School of Chemistry, Australian National University, \\ Canberra, Australian Capital Territory, 0200, Australia
}

(Dated: May 28, 2022)

\begin{abstract}
We prove that, in the large-dimension limit, the high-density correlation energy $E_{\mathrm{c}}$ of two oppositespin electrons confined in a $D$-dimensional space and interacting via a Coulomb potential is given by $E_{\mathrm{c}} \sim-1 /\left(8 D^{2}\right)$ for any radial confining potential $V(r)$. This result explains the observed similarity of $E_{\mathrm{c}}$ in a variety of two-electron systems in three-dimensional space.
\end{abstract}

PACS numbers: 31.15.ac, 31.15.ve, 31.15.xp, 31.15.xr, 31.15.xt

Keywords: correlation energy, two-electron systems, high-density limit, large-dimension limit

Understanding and calculating the electronic correlation energy is one of the most important and difficult problems in molecular physics. In this pursuit, the study of high-density correlation energy using perturbation theory has been particularly profitable, shedding light on the physically relevant density regime and providing exact results for key systems, such as the uniform electron gas [1] and two-electron systems [2]. The former is the cornerstone of the most popular density functional paradigm (the local density approximation) in solid-state physics [3]; the latter provide important test cases in the development of new explicitly correlated methods [4, 5, for electronic structure calculations [6]. Atomic units are used throughout.

The high-density correlation energy of the helium-like ions is obtained by expanding both the exact [7] and Hartree-Fock (HF) 8 energies as series in $1 / Z$, yielding

$$
\begin{aligned}
E(Z, D, V) & =E^{(0)}(D, V) Z^{2}+E^{(1)}(D, V) Z \\
& +E^{(2)}(D, V)+\frac{E^{(3)}(D, V)}{Z}+\ldots, \\
E_{\mathrm{HF}}(Z, D, V) & =E^{(0)}(D, V) Z^{2}+E^{(1)}(D, V) Z \\
& +E_{\mathrm{HF}}^{(2)}(D, V)+\frac{E_{\mathrm{HF}}^{(3)}(D, V)}{Z}+\ldots,
\end{aligned}
$$

where $Z$ is the nuclear charge, $D$ is the dimension of the space and $V$ is the external Coulomb potential. Equations (1) and (2) share the same zeroth- and first-order energies because the exact and the HF treatment have the same zeroth-order Hamiltonian. Thus, in the highdensity (large- $Z$ ) limit, the correlation energy is

$$
\begin{aligned}
E_{\mathrm{c}}^{(2)}(D, V) & =\lim _{Z \rightarrow \infty} E_{\mathrm{c}}(Z, D, V) \\
& =\lim _{Z \rightarrow \infty}\left[E(Z, D, V)-E_{\mathrm{HF}}(Z, D, V)\right] \\
& =E^{(2)}(D, V)-E_{\mathrm{HF}}^{(2)}(D, V) .
\end{aligned}
$$

Despite intensive study [9, 10, the coefficient $E^{(2)}(D, V)$ has not yet been reported in closed form. However, the accurate numerical estimate

$$
E^{(2)}=-0.15766642946914
$$

has been determined for the important $D=3$ case 10 . Combining (4) with the exact result 8

$$
E_{\mathrm{HF}}^{(2)}=\frac{9}{32} \ln \frac{3}{4}-\frac{13}{432}
$$

yields a value of

$$
E_{\mathrm{c}}^{(2)}=-0.04666325399948
$$

for the helium-like ions in 3-dimensional space.

In the large- $D$ limit, the quantum world reduces to a simpler semi-classical one [1] and problems that defy solution in $D=3$ sometimes become exactly solvable. In favorable cases, such solutions provide useful insight into the $D=3$ case and this strategy has been successfully applied in many fields of physics [12, 13. Indeed, just as one learns something about interacting systems by studying non-interacting ones and introducing the interaction perturbatively, one learns something about $D=3$ by studying the large- $D$ case and introducing dimensionreduction perturbatively.

Singularity analysis [14 reveals that the energies of two-electron atoms possess first- and second-order poles at $D=1$, and that the Kato cusp [15, 16 is directly responsible for the second-order pole. In our previous work [17, 18, we have expanded the correlation energy as a series in $1 /(D-1)$ but, although this is formally correct if summed to infinite order, such expansions falsely imply higher-order poles at $D=1$. For this reason, we now follow Herschbach and Goodson [19, 20], and expand both the exact and $\mathrm{HF}$ energies as series in $1 / D$. Although various possibilities exist for this dimensional expansion [14, 21, 23, it is convenient to write

$$
\begin{aligned}
& E^{(2)}(D, V)=\frac{E^{(2,0)}(V)}{D^{2}}+\frac{E^{(2,1)}(V)}{D^{3}}+\ldots, \\
& E_{\mathrm{HF}}^{(2)}(D, V)=\frac{E_{\mathrm{HF}}^{(2,0)}(V)}{D^{2}}+\frac{E_{\mathrm{HF}}^{(2,1)}(V)}{D^{3}}+\ldots, \\
& E_{\mathrm{c}}^{(2)}(D, V)=\frac{E_{\mathrm{c}}^{(2,0)}(V)}{D^{2}}+\frac{E_{\mathrm{c}}^{(2,1)}(V)}{D^{3}}+\ldots
\end{aligned}
$$


TABLE I. $E^{(2,0)}, E_{\mathrm{HF}}^{(2,0)}, E_{\mathrm{c}}^{(2,0)}$ and $E_{\mathrm{c}}^{(2,1)}$ coefficients for various systems and $v(r)=1$.

\begin{tabular}{lrcccc}
\hline \hline System & $m$ & $-E^{(2,0)}$ & $-E_{\mathrm{HF}}^{(2,0)}$ & $-E_{\mathrm{c}}^{(2,0)}$ & $-E_{\mathrm{c}}^{(2,1)}$ \\
\hline Helium & -1 & $5 / 8$ & $1 / 2$ & $1 / 8$ & 0.424479 \\
Airium & 1 & $7 / 24$ & $1 / 6$ & $1 / 8$ & 0.412767 \\
Hookium & 2 & $1 / 4$ & $1 / 8$ & $1 / 8$ & 0.433594 \\
Quartium & 4 & $5 / 24$ & $1 / 12$ & $1 / 8$ & 0.465028 \\
Sextium & 6 & $3 / 16$ & $1 / 16$ & $1 / 8$ & 0.486771 \\
Ballium & $\infty$ & $1 / 8$ & 0 & $1 / 8$ & 0.664063 \\
\hline \hline
\end{tabular}

where

$$
\begin{aligned}
& E_{\mathrm{c}}^{(2,0)}(V)=E^{(2,0)}(V)-E_{\mathrm{HF}}^{(2,0)}(V), \\
& E_{\mathrm{c}}^{(2,1)}(V)=E^{(2,1)}(V)-E_{\mathrm{HF}}^{(2,1)}(V) .
\end{aligned}
$$

Such double expansions of the correlation energy were originally introduced for the helium-like ions, and have lead to accurate estimations of correlation [24, 25] and atomic energies [26, 27] via interpolation and renormalization techniques. Equations (7), (8) and (9) apply equally to the ${ }^{1} S$ ground state of any two-electron system confined by a spherical potential $V(r)$.

For the helium-like ions, it is known [19, 20, 28, that

$$
E_{\mathrm{c}}^{(2,0)}(V)=-\frac{1}{8}, \quad E_{\mathrm{c}}^{(2,1)}(V)=-\frac{163}{384},
$$

and we have recently found [17 that $E_{\mathrm{c}}^{(2,0)}(V)$ takes the same value in hookium (two electrons in a parabolic well [29 32]), spherium (two electrons on a sphere 33 36]) and ballium (two electrons in a ball [18, 37, 38). In contrast, we found that $E_{\mathrm{c}}^{(2,1)}(V)$ is $V$-dependent. The fact that the term $E_{\mathrm{c}}^{(2,0)}$ is invariant, while $E_{\mathrm{c}}^{(2,1)}$ varies with the confinement potential allowed us to explain why the high-density correlation energy of the previous twoelectron systems are similar, but not identical, for $D=3$ [17, 18. On this basis, we conjectured [17] that

$$
E_{\mathrm{c}}^{(2)}(D, V) \sim-\frac{1}{8 D^{2}}-\frac{C(V)}{D^{3}}
$$

holds for any spherical confining potential, where the coefficient $C(V)$ varies slowly with $V(r)$.

In this Letter, we prove that $E_{\mathrm{c}}^{(2,0)}$ is indeed universal, and that, in the large- $D$ limit, the high-density correlation energy of the ${ }^{1} S$ ground state of two electrons is given by (13) for any confining potential of the form

$$
V(r)=\operatorname{sgn}(m) r^{m} v(r),
$$

where $v(r)$ possesses a Maclaurin series expansion

$$
v(r)=v_{0}+v_{1} r+v_{2} r^{2}+\ldots
$$

In order to prove the conjecture [13], we start with the conventional Schrödinger equation

$$
\hat{H} \Psi_{D}=E_{D} \Psi_{D},
$$

and the general Hamiltonian

$$
\hat{H}=-\frac{1}{2}\left(\nabla_{1}^{2}+\nabla_{2}^{2}\right)+Z^{m+2}\left[V\left(r_{1}\right)+V\left(r_{2}\right)\right]+\frac{1}{r_{12}},
$$

where $Z$ is the confinement strength and $r_{12}=\left|\boldsymbol{r}_{1}-\boldsymbol{r}_{2}\right|$ is the interelectronic distance. After the Jacobianweighted transformation

$$
\begin{gathered}
\Phi_{D}=\mathcal{J}^{1 / 2} \Psi_{D}, \\
\mathcal{J}=r_{1}^{D-1} r_{2}^{D-1} \sin ^{D-2} \theta,
\end{gathered}
$$

where $\theta$ is the interelectronic angle, the Schrödinger equation (16) becomes

$$
\left(\hat{\mathcal{T}}+\Lambda \hat{\mathcal{U}}+Z^{m+2} \hat{\mathcal{V}}+\hat{\mathcal{W}}\right) \Phi_{D}=E_{D} \Phi_{D}
$$

in which, for states with zero total angular momentum, the kinetic, centrifugal, external and Coulomb operators are respectively

$$
\begin{gathered}
-2 \hat{\mathcal{T}}=\left(\frac{\partial^{2}}{\partial r_{1}^{2}}+\frac{\partial^{2}}{\partial r_{2}^{2}}\right)+\left(\frac{1}{r_{1}^{2}}+\frac{1}{r_{1}^{2}}\right)\left(\frac{\partial^{2}}{\partial \theta^{2}}+\frac{1}{4}\right), \\
\hat{\mathcal{U}}=\frac{1}{2 \sin ^{2} \theta}\left(\frac{1}{r_{1}^{2}}+\frac{1}{r_{1}^{2}}\right), \\
\hat{\mathcal{V}}=V\left(r_{1}\right)+V\left(r_{2}\right) \\
\hat{\mathcal{W}}=\frac{1}{\sqrt{r_{1}^{2}+r_{2}^{2}-2 r_{1} r_{2} \cos \theta}}
\end{gathered}
$$

and

$$
\Lambda=\frac{(D-2)(D-4)}{4} .
$$

We now need to recast the Schrödinger equation so that perturbation theory can be applied. To achieve this, we successively introduce the scaled quantities

$$
r \rightarrow \frac{\Lambda}{\kappa Z} r, \quad Z \rightarrow \frac{Z}{\kappa},
$$

where $\kappa=\Lambda^{\frac{m+1}{m+2}}$, and introduce the scaled energy

$$
\mathcal{E}_{D}=\frac{\kappa^{2} Z^{2}}{\Lambda} E_{D}
$$

The Schrödinger equation then takes the simple form

$$
\left(\frac{1}{\Lambda} \hat{\mathcal{T}}+\hat{\mathcal{U}}+\hat{\mathcal{V}}+\frac{1}{Z} \hat{\mathcal{W}}\right) \Phi_{D}=\mathcal{E}_{D} \Phi_{D}
$$

and it is clear that perturbation theory can now be used to expand the energy both in terms of $Z$ and $\Lambda$. 
In the $D=\infty$ limit, the kinetic term vanishes and classical electrostatics cause the electrons to settle into a fixed ("Lewis") structure [19] that minimizes the effective potential

$$
\hat{\mathcal{X}}=\hat{\mathcal{U}}+\hat{\mathcal{V}}+\frac{1}{Z} \hat{\mathcal{W}}
$$

The minimization conditions are

$$
\begin{gathered}
\frac{\partial \hat{\mathcal{X}}\left(r_{1}, r_{2}, \theta\right)}{\partial r_{1}}=\frac{\partial \hat{\mathcal{X}}\left(r_{1}, r_{2}, \theta\right)}{\partial r_{2}}=0, \\
\frac{\partial \hat{\mathcal{X}}\left(r_{1}, r_{2}, \theta\right)}{\partial \theta}=0
\end{gathered}
$$

and the stability condition implies $m>-2$. Assuming that the two electrons are equivalent [39], the resulting exact density and energy are

$$
\begin{gathered}
\left|\Phi_{\infty}\right|^{2}=\delta\left(r_{1}-r_{\infty}\right) \delta\left(r_{2}-r_{\infty}\right) \delta\left(\theta-\theta_{\infty}\right), \\
\mathcal{E}_{\infty}=\hat{\mathcal{X}}\left(r_{\infty}, r_{\infty}, \theta_{\infty}\right)
\end{gathered}
$$

where $\delta$ is the Dirac delta function. Substituting Taylor expansions of $r_{\infty}$ and $\theta_{\infty}$ into 30 and 31 yields

$$
\begin{gathered}
r_{\infty}=\alpha+\frac{\alpha^{2}}{m+2}\left(\frac{1}{2 \sqrt{2}}-\Lambda \frac{m+1}{m} \frac{v_{1}}{v_{0}}\right) \frac{1}{Z}+\ldots \\
\cos \theta_{\infty}=-\frac{\alpha}{4 \sqrt{2}} \frac{1}{Z}+\ldots
\end{gathered}
$$

where $\alpha^{-(m+2)}=\operatorname{sgn}(m) m v_{0}$. The $m=0$ case requires special attention, and is found by taking the $m \rightarrow 0$ limit.

For the HF energy, things are simpler. The HF wave function is independent of $\theta$, so the only angular dependence comes from the Jacobian 19. Moreover, because

$$
\lim _{D \rightarrow \infty} \frac{\sin ^{D-2} \theta}{\int_{0}^{\pi} \sin ^{D-2} \theta d \theta}=\delta\left(\theta-\frac{\pi}{2}\right),
$$

it follows [20] that $\theta_{\infty}^{\mathrm{HF}}=\pi / 2$. Solving (30), one finds that $r_{\infty}^{\mathrm{HF}}$ and $r_{\infty}$ are equal to second-order in $1 / Z$. Thus, in the large- $D$ limit, the HF density and energy are

$$
\begin{gathered}
\left|\Phi_{\infty}^{\mathrm{HF}}\right|^{2}=\delta\left(r_{1}-r_{\infty}^{\mathrm{HF}}\right) \delta\left(r_{2}-r_{\infty}^{\mathrm{HF}}\right) \delta\left(\theta-\frac{\pi}{2}\right) \\
\mathcal{E}_{\infty}^{\mathrm{HF}}=\hat{\mathcal{X}}\left(r_{\infty}^{\mathrm{HF}}, r_{\infty}^{\mathrm{HF}}, \frac{\pi}{2}\right)
\end{gathered}
$$

and correlation effects originate entirely from the fact that $\theta_{\infty}$ is slightly greater than $\pi / 2$ for finite $Z$.

Expanding (33) and 38 in terms of $Z$ and $D$ yields

$$
\begin{aligned}
& E^{(2,0)}(V)=-\frac{1}{8}-\frac{1}{2(m+2)}, \\
& E_{\mathrm{HF}}^{(2,0)}(V)=-\frac{1}{2(m+2)},
\end{aligned}
$$

thus showing that both $E^{(2,0)}$ and $E_{\mathrm{HF}}^{(2,0)}$ depend on the leading power $m$ of the external potential but not on $v(r)$.

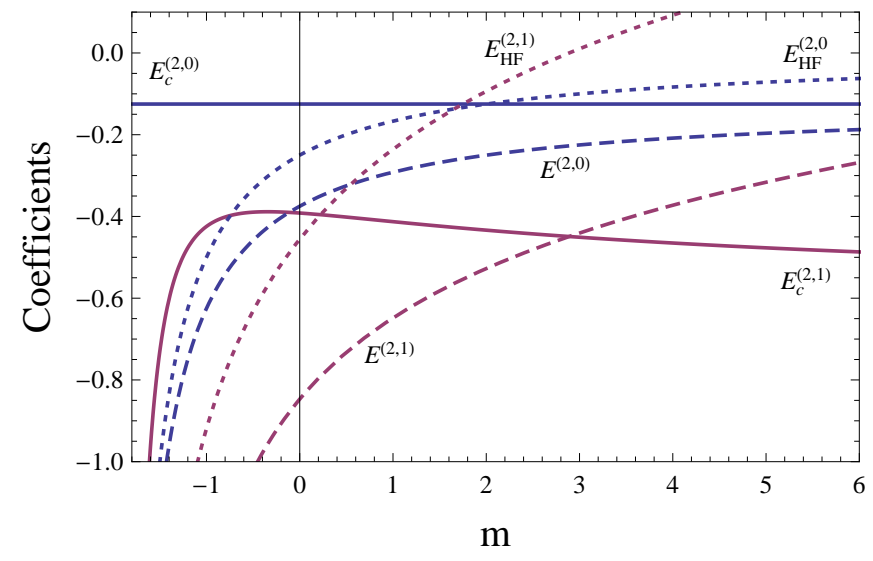

FIG. 1. Coefficients of the exact (dashed), HF (dotted) and correlation (solid) energies with respect to $m$, for $v(r)=1$ (Eqs. 7), 8) and (9).

Subtracting these energies yields

$$
E_{\mathrm{c}}^{(2,0)}(V)=-\frac{1}{8}
$$

and completes the proof that, in the high-density limit, the leading coefficient $E_{\mathrm{c}}^{(2,0)}$ of the large- $D$ expansion of the correlation energy is universal, i.e. it does not depend on the external potential $V(r)$.

What is the origin of the constant in Eq. 411)? It comes directly from the leading coefficient $(1 / 4 \sqrt{2})$ in the $1 / Z$ expansion of $\theta_{\infty}$ (Eq. (35) ) and, because that is determined via Eq. 31, it is independent of the external potential $V(r)$. This reveals that Eq. (41) applies to a pair of electrons in any radial external potential, but not to anisotropic external potentials.

Detailed analysis of $E_{\mathrm{c}}^{(2,0)}$ shows that it results from contributions of $+1 / 8$ and $-1 / 4$ from the centrifugal potential $\hat{\mathcal{U}}$ and the Coulomb operator $\hat{\mathcal{W}}$, respectively. The external potential $\hat{\mathcal{V}}$, which contributes identically in the exact and $\mathrm{HF}$ treatments, does not contribute to the correlation energy. Kato has made a similar argument [15] to explain the behavior of the wave function as $r_{12} \rightarrow 0$. In a $D$-dimensional space, the Kato cusp condition is [16]

$$
\left.\frac{\partial \Psi_{D}}{\partial r_{12}}\right|_{r_{12}=0}=\frac{1}{D-1} \Psi_{D}\left(r_{12}=0\right),
$$

and arises from the cancelation of the singularities in the Coulomb operator and the $D$-dependent angular part of the kinetic operator [6]. These observations suggest a connection between the result (41) and the Kato cusp (42). For large but finite $D$, the discovery that the Kato cusp plays a key role in the large- $Z$ limit would not be surprising for, in this limit, the only relevant information is the behavior (42) of the wave function near $r_{12}=0$.

The $E^{(2,1)}$ and $E_{\mathrm{HF}}^{(2,1)}$ coefficients can be found by considering the Langmuir vibrations of the electrons around 
their equilibrium positions [19, 20]. The general expressions depend on $v_{0}$ and $v_{1}$, but are not reported here. However, for $v(r)=1$, which includes many of the most common external potentials, we find

$$
\begin{aligned}
E_{\mathrm{c}}^{(2,1)}(V)=- & \frac{85}{128}-\frac{9 / 32}{(m+2)^{3 / 2}} \\
& +\frac{1 / 2}{(m+2)^{1 / 2}}+\frac{1 / 16}{(m+2)^{1 / 2}+2}
\end{aligned}
$$

showing that $E_{\mathrm{c}}^{(2,1)}$, unlike $E_{\mathrm{c}}^{(2,0)}$, is potential-dependent. It is singular at $m=-2$, tends to $-85 / 128$ as $m \rightarrow \infty$, and reaches a maximum of -0.388482 at $m \approx-0.344223$. The latter value of $m$ corresponds to the minimum of the correlation energy in the large- $D$ limit. Numerical values of $E_{\mathrm{c}}^{(2,1)}$ are reported in Table $\mathrm{I}$ for various systems, and the components of the correlation energy are shown graphically in Fig. 1 .

In conclusion, we have proved that the leading term $E_{\mathrm{c}} \sim-1 /\left(8 D^{2}\right)$ in the large- $D$ expansion of the highdensity correlation energy of an electron pair is invariant to the nature of the radial confining potential. Although formally divergent 40, truncated $1 / D$ expansions have been found to be a powerful tool for the exploration of correlation effects and, in the present study, they help to explain the observation that, in finite-dimensional spaces such as $D=3$, the correlation energy depends only weakly on the confining potential.

We thank Andrew Gilbert for several stimulating discussions at the early stage of this work. P.M.W.G. thanks the NCI National Facility for a generous grant of supercomputer time and the Australian Research Council (Grants DP0771978 and DP0984806) for funding.

* loos@rsc.anu.edu.au

$\dagger$ Corresponding author; peter.gill@anu.edu.au

[1] M. Gell-Mann and K. A. Brueckner, Phys. Rev. 106, 364 (1957).

[2] H. A. Bethe and E. E. Salpeter, Quantum Mechanics of One- and Two-Electron Atoms (Dover Publications Inc., Mineola, New-York, 1977).

[3] R. G. Parr and W. Yang, Density Functional Theory for Atoms and Molecules (Oxford University Press, 1989).

[4] W. Kutzelnigg, Theor. Chim. Acta 68, 445 (1985).

[5] H. Nakashima and H. Nakatsuji, J. Chem. Phys. 127, 224104 (2007).

[6] T. Helgaker, P. Jørgensen, and J. Olsen, Molecular Electronic-Structure Theory (John Wiley \& Sons, Ltd., 2000).

[7] E. A. Hylleraas, Z. Phys. 65, 209 (1930).

[8] J. Linderberg, Phys. Rev. 121, 816 (1961).

[9] C. Schwartz, Phys. Rev. 126, 1015 (1962).
[10] J. D. Baker, D. E. Freund, R. N. Hill, and J. D. Morgan III, Phys. Rev. A 41, 1247 (1990).

[11] L. G. Yaffe, Rev. Mod. Phys. 54, 407 (1982).

[12] E. Witten, Physics Today 33, 38 (1980).

[13] L. G. Yaffe, Physics Today 36, 50 (1983).

[14] D. J. Doren and D. R. Herschbach, J. Chem. Phys. 87, 443 (1987).

[15] T. Kato, Commun. Pure Appl. Math. 10, 151 (1957).

[16] J. D. Morgan III, The dimensional dependence of rates of convergence of Rayleigh-Ritz variational calculations on atoms and molecules (Kluwer Academic Publishers, Dordrecht, 1993), p. 336, Dimensional Scaling in Chemical Physics.

[17] P.-F. Loos and P. M. W. Gill, J. Chem. Phys. 131, 241101 (2009).

[18] P.-F. Loos and P. M. W. Gill, J. Chem. Phys. 132, 234111 (2010).

[19] D. R. Herschbach, J. Chem. Phys. 84, 838 (1986).

[20] D. Z. Goodson and D. R. Herschbach, J. Chem. Phys. 86, 4997 (1987).

[21] D. J. Doren and D. R. Herschbach, Phys. Rev. A 34, 2654 (1986).

[22] D. Z. Goodson, M. López-Cabrera, D. R. Herschbach, and J. D. Morgan III, J. Chem. Phys 97, 8481 (1992).

[23] D. Z. Goodson and M. López-Cabrera, Low-D regime: the one-dimensional limit (Kluwer Academic Publishers, Dordrecht, 1993), p. 115, Dimensional Scaling in Chemical Physics.

[24] J. G. Loeser and D. R. Herschbach, J. Chem. Phys. 86, 2114 (1987).

[25] J. G. Loeser and D. R. Herschbach, J. Chem. Phys. 86, 3512 (1987).

[26] J. G. Loeser, J. Chem. Phys. 86, 5635 (1987).

[27] S. Kais, S. M. Sung, and D. R. Herschbach, J. Chem. Phys 99, 5184 (1993).

[28] L. D. Mlodinow and N. Papanicolaou, Ann. Phys. 131, 1 (1981).

[29] N. R. Kestner and O. Sinanoglu, Phys. Rev. 128, 2687 (1962).

[30] R. J. White and W. Byers Brown, J. Chem. Phys. 53, 3869 (1970).

[31] S. Kais, D. R. Herschbach, and R. D. Levine, J. Chem. Phys 91, 7791 (1989).

[32] M. Taut, Phys. Rev. A 48, 3561 (1993).

[33] G. S. Ezra and R. S. Berry, Phys. Rev. A 25, 1513 (1982).

[34] M. Seidl, Phys. Rev. A 75, 062506 (2007).

[35] P.-F. Loos and P. M. W. Gill, Phys. Rev. A 79, 062517 (2009).

[36] P.-F. Loos and P. M. W. Gill, Phys. Rev. Lett. 103, 123008 (2009).

[37] D. C. Thompson and A. Alavi, Phys. Rev. B 66, 235118 (2002).

[38] D. C. Thompson and A. Alavi, J. Chem. Phys. 122, 124107 (2005).

[39] In the helium atom, for $Z<Z_{\text {crit }}\left(Z_{\text {crit }} \approx 1.228\right.$ and $\left.Z_{\mathrm{crit}}^{\mathrm{HF}} \approx 0.8839\right)$, the configuration $r_{1}=r_{2}$ becomes a saddle point between two minima corresponding to nonsymmetric configurations [19, 20]. This is irrelevant, however, in the high-density regime.

[40] M. O. Elout, D. Z. Goodson, C. D. Elliston, S.-W. Huang, A. V. Sergeev, and D. K. Watson, J. Math. Phys. 39, 5112 (1998). 